\title{
Secondary flow and heat transfer coefficient distributions in the developing flow region of ribbed turbine blade cooling passages
}

\author{
Peter Forsyth $^{1}$ (1) $\cdot$ Matthew McGilvray $^{1}$ David R. H. Gillespie ${ }^{1}$
}

Received: 18 January 2016 / Revised: 29 November 2016 / Accepted: 29 November 2016 / Published online: 19 December 2016

(C) The Author(s) 2016. This article is published with open access at Springerlink.com

\begin{abstract}
This paper reports an experimental and numerical study of the development and coupling of aerodynamic flows and heat transfer within a model ribbed internal cooling passage to provide insight into the development of secondary flows. Static instrumentation was installed at the end of a long smooth passage and used to measure local flow features in a series of experiments where ribs were incrementally added upstream. This improves test turnaround time and allows higher-resolution heat transfer coefficient distributions to be captured, using a hybrid transient liquid crystal technique. A composite heat transfer coefficient distribution for a 12-rib-pitch passage is reported: notably the behaviour is dominated by the development of the secondary flow in the passage throughout. Both the aerodynamic and heat transfer test data were compared to numerical simulations developed using a commercial computational fluid dynamics solver. By conducting a number of simulations it was possible to interrogate the validity of the underlying assumptions of the experimental strategy; their validity is discussed. The results capture the developing size and strength of the vortical structures in secondary flow. The local flow field was shown to be strongly coupled to the enhancement of heat transfer coefficient. Comparison of the experimental and numerical data generally shows excellent agreement in the level of heat transfer coefficient predicted, though the numerical simulations fail to capture some local enhancement on both the ribbed and
\end{abstract}

First presented at ASME IGTI Dusseldorf 2014 as paper GT2014-26737.

Peter Forsyth

peter.forsyth@eng.ox.ac.uk

1 Osney Thermofluids Laboratory, Department of Engineering Science, University of Oxford, Oxford OX2 0ES, UK smooth surfaces. Where this was the case, the coupled flow and heat transfer measurements were able to identify missing velocity field characteristics.

$\begin{array}{ll}\text { List of } & \text { symbols } \\ A_{x} & \text { Cross-sectional area }\left(\mathrm{m}^{2}\right) \\ c_{p} & \text { Specific heat capacity }(\mathrm{J} / \mathrm{kg} / \mathrm{K}) \\ D_{\mathrm{h}} & \text { Hydraulic diameter }(\mathrm{m}) \\ e & \text { Rib height }(\mathrm{m}) \\ k & \text { Thermal conductivity }(\mathrm{W} / \mathrm{m} / \mathrm{K}) \\ P & \text { Pressure }(\mathrm{Pa}) \\ p & \text { Rib pitch }(\mathrm{m}) \\ \mathrm{Nu} & \text { Nusselt number }\left(\frac{h D_{\mathrm{h}}}{k}\right) \\ \dot{q} & \text { Heat flux }\left(\mathrm{W} / \mathrm{m}^{2}\right) \\ \mathrm{Re} & \text { Reynolds number }\left(\frac{\rho u D_{\mathrm{h}}}{\mu}\right) \\ T & \text { Temperature }(\mathrm{K}) \\ t & \text { Time }(\mathrm{s}) \\ u & \text { Gas velocity }(\mathrm{m} / \mathrm{s})\end{array}$

Greek

$\alpha \quad$ Rib angle $\left({ }^{\circ}\right)$

$\mu \quad$ Viscosity (Pa s)

$\rho \quad$ Density $\left(\mathrm{kg} / \mathrm{m}^{3}\right)$

\begin{abstract}
Abbreviations
AR Aspect ratio (width/height)

CFD Computational fluid dynamics

HTC, $h \quad$ Heat transfer coefficient $\left(\mathrm{W} / \mathrm{m}^{2} / \mathrm{K}\right)$
\end{abstract}

\section{Introduction}

High-pressure turbine blades and vanes require cooling using colder gas bled from the compressor to decrease metal temperatures. Common methods include external 
film cooling, internal impingement cooling and rib-turbulated cooling. The engine designer must trade-off improved efficiency and specific power of the engine achieved through higher combustor exit temperatures against the potential reduction in component life. Care must be taken to minimise the mass flow rate of coolant used as it reduces the potential enthalpy change available for thrust generation (Saha and Acharaya 2011). Inefficient use of coolant can limit the maximum efficiency that can be achieved (Horlock et al. 2001). Rib-turbulated cooling is commonly applied by designers in the mid-chord region of turbine blades. Higher heat transfer coefficients are achieved by placing a ridge, or rib, angled to the bulk direction of the flow. This disrupts the incoming boundary layer causing a small separation behind the rib. Downstream, the boundary layer restarts resulting in a higher heat transfer. Mixing is enhanced by interaction of the secondary flows, induced by the inclined ribs, and the mainstream flow, while local turbulence increases mixing in the weak areas of the ribs.

Flow development is commonly ignored in the literature and more commonly is assumed to reach a steady, fully developed state (Lau et al. 1991; Liou et al. 1993). However, a considerable length of internal cooling passages is in fact in the initial developing region (typically up to half the span). Fully developed assumptions have allowed comprehensive empirical correlations to be created and used in cooling designs to approximate the wall heat transfer (Han et al. 1989, 2000). With numerical calculations routinely used in design and optimisation, engineers need to verify simulations across the full passage. Thus, further data are required for verification and understanding of this process.

There have been numerous studies of the heat transfer of ribbed turbulated passages and others on the detailed aerodynamics (e.g. Han et al. 1989, 2000; Tanda 2010). However, only a few have explicitly linked the aerodynamics within the passage to the spatial distributions of heat transfer coefficient (Rau et al. 1998; Casarsa and Arts 2005; Arts 2006). Some (Casarsa and Arts 2005; Iacovides and Launder 2007) discuss the importance of combined aerodynamic and heat transfer data. For true numerical code validation, combined studies are required to fully understand why discrepancies are occurring, and where detailed flow features are not captured. Such detail is required to provide robust codes suitable for automated optimisation of design.

This paper introduces an experimental methodology to combine aerodynamic measurements and spatial measurements of heat transfer at various stages of flow development and allow rapid reconfiguration of rib/passage parameters. Results are presented for a Reynolds number of 100,000, based on smooth passage hydraulic diameter, in a ribturbulated passage with a 1:2 aspect ratio. Accompanying numerical simulations are compared to the experimental measurements and provide insight into where the numerical models fail to fully capture the turbulent/physical processes correctly in the calculations.

\section{Experimental rig and passage design}

The premise of the experimental rig was to allow combined aerodynamic and heat transfer testing at different stages of secondary flow development. The rig design allows different rib geometry properties $\left(e / D_{\mathrm{h}}\right.$, angle, $p / e$, etc. $)$ to be rapidly altered. The experimental passage was approximately 20 times engine scale and allows aerodynamic measurements to be taken using a blockage-resistant fourhole probe. This large scale also allowed for high spatial resolution heat transfer data to be gathered by applying the transient liquid crystal technique. The cooling passage is simplified to a rectangular geometry, Fig. 1 .

The test section is installed in a low-speed wind tunnel to model a ribbed cooling passage. The tunnel has an atmospheric intake and is driven by an axial fan at exit. Flow is conditioned at the entrance to the tunnel using a 10:1 area contraction, and small-scale turbulence is removed through the inclusion of a heater mesh upstream of the test section. There is a $500 \times 150 \times 150 \mathrm{~mm}$ square section passage between the heater mesh and flow contraction; this is shortened in Fig. 1. The rig is instrumented with an aerodynamic probe driven by a traverse mechanism. A multiple liquid crystal coating is applied over the final three rib pitches at the exit of the test

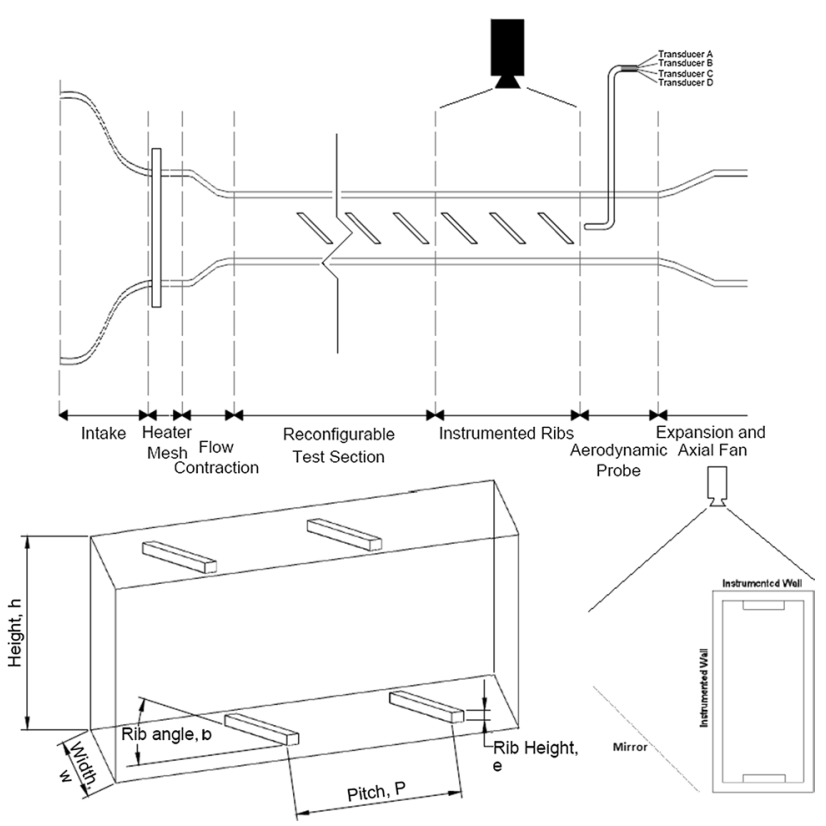

Fig. 1 Rig layout. Clockwise from top: top view of passage; passage cross section; schematic layout of test section. Not to scale 
section where pairs of ribs are permanently fixed to the walls of the passage. By adding ribs upstream of these in each subsequent test, measurements can be made for any streamwise position in the passage. When only a single rib is in place, there is a $1500 \mathrm{~mm}$ upstream flow development length, significantly larger than the traditional rule of $10 D_{\mathrm{h}}$ (Kreith and Boehm 2000). After this first rib the boundary layer profile on the ribbed walls and leading edge was significantly altered by the presence of the ribs; this was observed in our simulations. The engine-representative regions of heat transfer from each test were then combined to form a full heat transfer coefficient distribution for the entire passage length (up to the 12 rib pitches investigated).

This new method for fast, combined aerodynamic and heat transfer experimental work has some advantages over existing techniques. The primary advantage is that geometric adjustments can be undertaken quickly, allowing multiple geometries to be tested experimentally: however, the area investigated is not reinstrumented making the technique faster. The resolution of heat transfer coefficient is high, because of the small area recorded in each test: experimental work undertaken for this paper recorded data at 3.7 times (suction surface) and 2.1 times (leading edge) resolutions typical for traditional full passage testing (Ryley et al. 2013). This significantly higher resolution has substantial advantages, including more reliable processing and improved viewing in areas of high heat flux gradient.

Another key advantage is that the super-scaling of the model allowed aerodynamic measurements to be taken using a large blockage-resistant probe. By increasing numbers of ribs in subsequent experiments multiple cross-sectional velocity distributions are measured, which was not possible non-invasively in previous experimental work. These cross sections of the developing flow have been essential in providing explanation of the development of the flow and the differences seen between experimental and numerically simulated heat transfer.

\subsection{Ribbed passage}

The test section (Fig. 1) has a rectangular cross section, with an aspect ratio of 1:2 (width/height). The cross section is of dimension $75 \times 150 \mathrm{~mm}$ and length $1400 \mathrm{~mm}$. The hydraulic diameter $\left(D_{\mathrm{h}}\right)$ is $100 \mathrm{~mm}$. Ribs are spaced alternately at half-pitch intervals on the narrower surfaces, in parallel planes. The ribs are $6 \times 6 \mathrm{~mm}$ square sections and $53 \mathrm{~mm}$ in length, spanning the middle half of the passage. The ribbed passage geometry is: rib spacing $P / e=10$, blockage ratio $e / D_{\mathrm{h}}=6.0 \%$ and rib angle $\alpha=45^{\circ}$ to the streamwise direction.

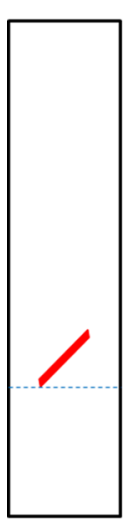

Case 1

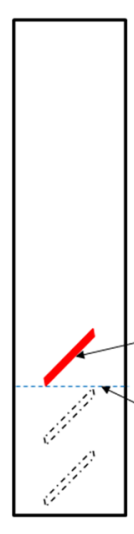

Case 2

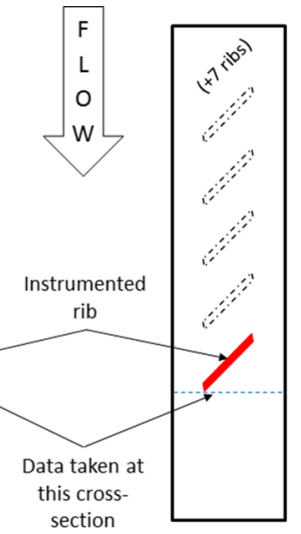

Case 3

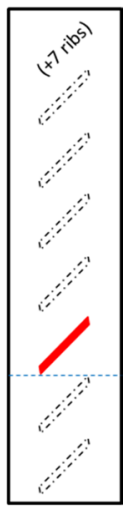

Case 4
Fig. 2 Rib configurations used for rig validation. The solid (red) rib lies immediately upstream of the measurement plane

\subsection{Validation of rig layout}

A series of numerical simulations were undertaken to demonstrate that fixing the position of the aerodynamic instrumentation and building upstream features was equivalent to taking measurements at different cross sections along a fixed passage with the same number of upstream ribs. These were undertaken using a simulation methodology and with similar mesh properties to those fully described below (Sect. 4).

Two comparisons are made, shown in Fig. 2, in order to assess the influence of ribs installed downstream of the measurement plane. In cases 1 and 2 a single rib is installed upstream of the measurement plane; in cases 3 and 4, 11 ribs (four shown, plus seven unseen) are upstream of the measurement plane. The differences case 1 to 2 and 3 to 4 are the presence of two rib sets downstream of the measurement plane. In Figs. 3 and 4, the upper row shows maps of pitch (vertical) angle and the lower row maps of yaw (horizontal) angle for both the cases compared, and the absolute difference between cases.

Comparisons are made only for the top half of the passage for the reasons detailed below. The pitch angle distribution in this region was reasonably similar in cases 1 and 2, with $83 \%$ of the cross-sectional area showing a difference of $< \pm 1^{\circ}$ and $96 \%< \pm 3^{\circ}$. The yaw angle shows slightly smaller differences: $88 \%$ of the cross-sectional lay $< \pm 1^{\circ}$ and $95 \%< \pm 3^{\circ}$.

For cases 3 and 4 the effect of the absence of downstream ribs on the pitch angle is more pronounced; $68 \%$ of the passage cross-sectional area now had $< \pm 1^{\circ}$ difference and $97 \%< \pm 3^{\circ}$. The match between yaw angle was again better than for pitch; $84 \%$ of the passage showing $< \pm 1^{\circ}$ difference and $97 \%< \pm 3^{\circ}$.

The conclusion drawn was that the upstream potential field caused by the extra downstream ribs in cases 2 and 4 


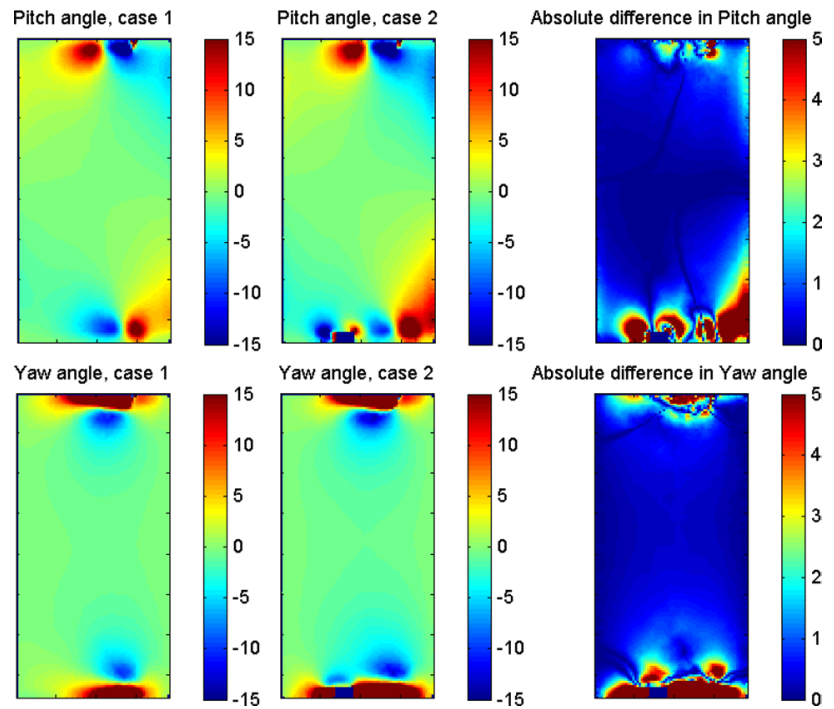

Fig. 3 Pitch and yaw angle distributions at defined cross section (as shown in Fig. 2), cases 1 and 2. Directions defined in Fig. 5

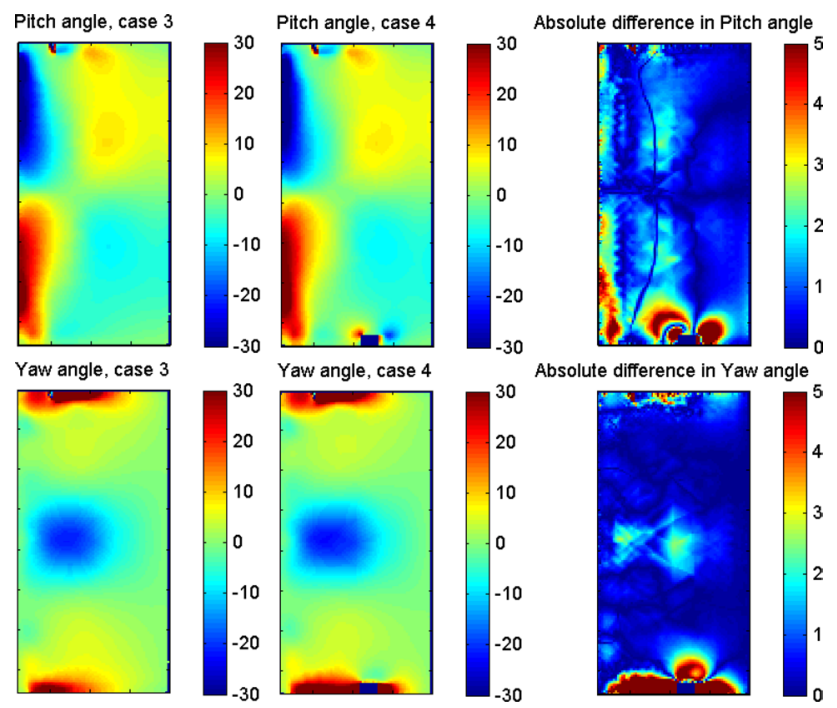

Fig. 4 Pitch and yaw angle distributions at defined cross section (as shown in Fig. 2), cases 3 and 4. Directions defined in Fig. 5

was small in the upper half of the passage. The lower half of the passage is discounted as the plane necessarily crosses through a rib, causing non-representative features in these regions in the figures from cases 2 and 4 that would not exist experimentally.

\section{Experimental test strategy}

Two tests were undertaken for each configuration of ribs installed (relating to increasingly developed flow in the

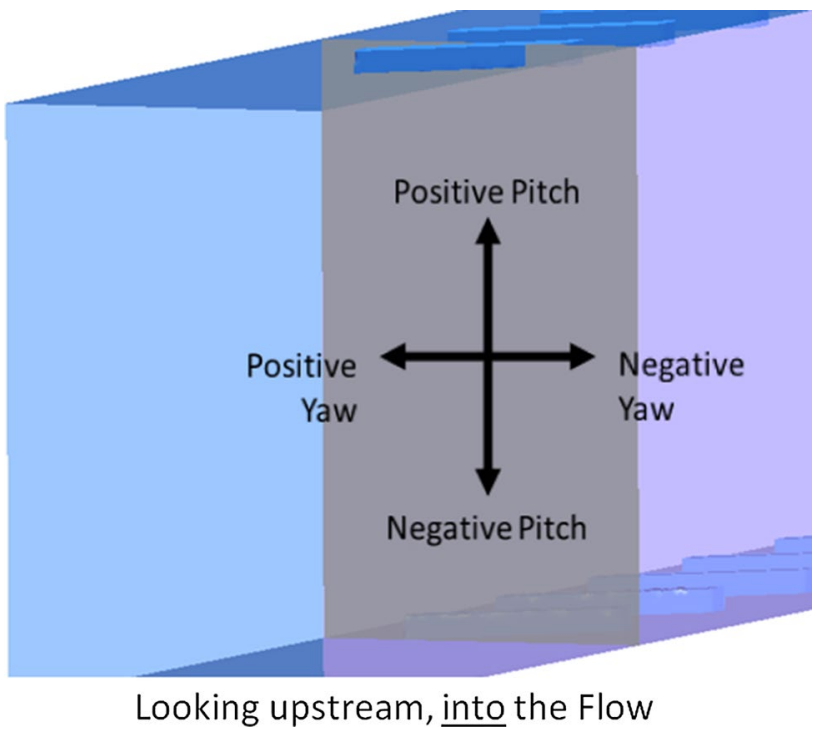

Fig. 5 Aerodynamic pitch and yaw directions

passage). In both cases tests were run at a target enginerepresentative Reynolds number of 100,000.

\subsection{Aerodynamic tests}

The first test was an aerodynamic test, employing a fourhole pyramid probe which was slowly traversed across the passage measurement plane. The measurement plane was divided into a $3 \times 3 \mathrm{~mm}$ grid of points at which aerodynamic data were measured. Aerodynamic tests were run with an unheated mainstream.

\subsection{Four-hole pyramid probe}

The four-hole probe mounted on a traverse mechanism used to measure pressures at discrete points within the flow at a fixed cross section of the passage is shown in Fig. 6. The pyramid probe design allows the pitch and yaw to be measured in addition to the dynamic head and total pressure (Main
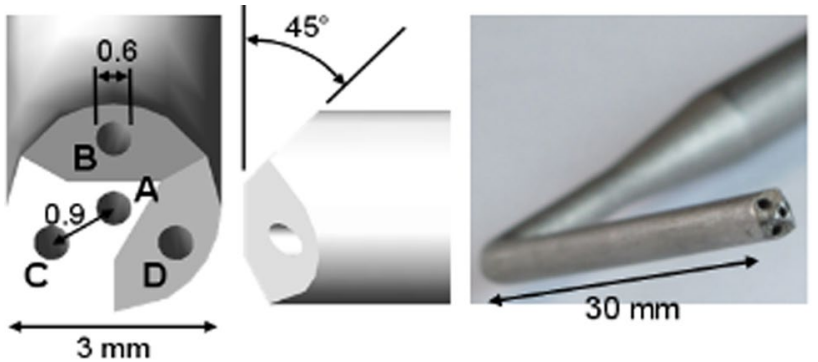

Fig. 6 Four-hole probe used for aerodynamic measurements (Kwan 2011) 


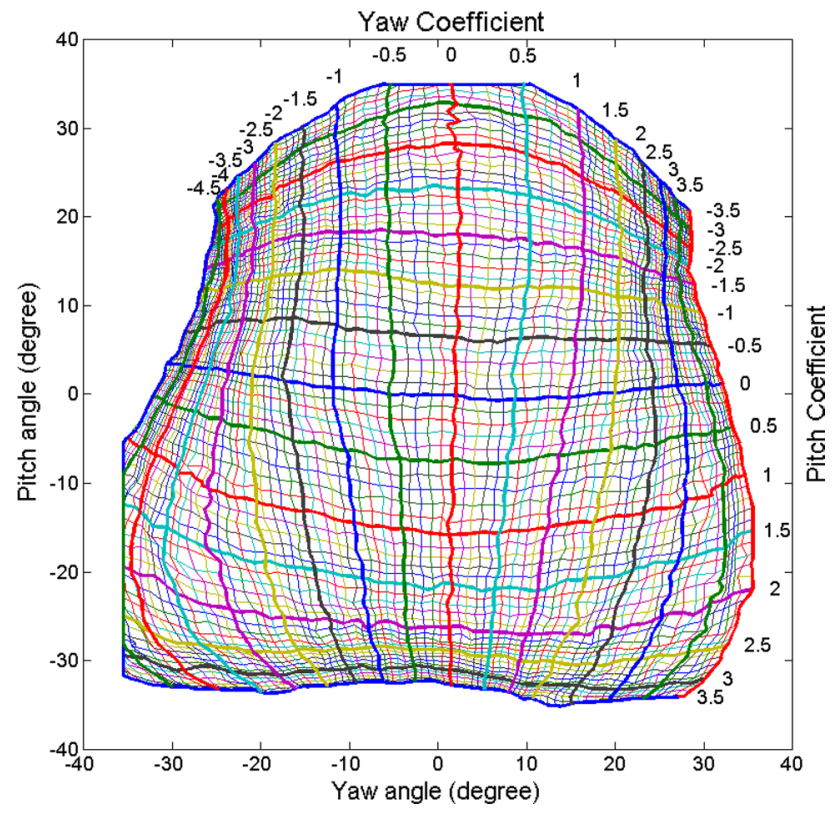

Fig. 7 Map of aerodynamic calibration. Labelled values within the plot indicate coefficient contour values

et al. 1996; Tsang and Oldfield 1996). When aligned with the streamwise direction and facing upstream, the central hole measures the stagnation pressure when the flow was fully streamwise. The three peripheral holes measured a combination of static and dynamic pressure. The sharp edges to the peripheral faces caused the flow to separate, making Reynolds effects negligible over the range of flow velocities investigated. Due to the low Mach number $(>0.05)$ compressible effects of the probe were considered negligible.

Probe pressures were used to calculate non-dimensional pitch, yaw and dynamic head coefficients using Eqs. 1-4. A calibration of the probe (Fig. 7) was carried out at known pitch and yaw angles from $-30^{\circ}$ to $+30^{\circ}$ in both axes. This allowed the relationship between angles and coefficients to be calculated as 2-D maps. By calculating the pitch and yaw coefficients from measured data a reverse lookup could then be achieved.

$$
\begin{aligned}
& C_{\text {pitch }}=\frac{P_{b}-\frac{1}{2}\left(P_{c}+P_{d}\right)}{P_{a}-P_{\text {mean }}} \\
& C_{\text {yaw }}=\frac{P_{c}-P_{d}}{P_{a}-P_{\text {mean }}} \\
& C_{\text {dyn }}=\frac{P_{d y n}}{P_{a}-P_{\text {mean }}} \\
& P_{\text {mean }}=\frac{P_{a}+P_{b}+P_{c}}{3}
\end{aligned}
$$

All pressure transducer signals were recorded by Measurement Computing CIO-DAS082/16 16-bit analogue to digital data acquisition board, which provided a pressure resolution of $0.19 \mathrm{~Pa}$ for the 0-50 mbar (Sensortecnics HCXM050D6V) transducers used to measure the static pressure tappings and $0.076 \mathrm{~Pa}$ for the 0-20 mbar transducer (Sensortecnics HCXM020D6V) used for the total tapping. Transducer accuracies were \pm 5.92 and $\pm 0.88 \mathrm{~Pa}$, respectively. This data acquisition board and the stepper motor controller board were installed on the same computer. The software which automated the traverse mechanism for the four-hole pyramid probe and data acquisition board was developed in the National Instrument LabVIEW 7.1 environment. Sufficient time was allowed for four-hole pyramid probe pressure measurements to become steady at each grid point: $5 \mathrm{~s}$ for the motion of the traverse, $1 \mathrm{~s}$ for pressure settling time within the four-hole pyramid probe and a 2.5-s sampling period. During each sampling period 156 samples were taken and averaged to produce a single data point. The sampling time period is significantly larger than that of any unsteadiness in the flow; hence, it is assumed that sampling will integrate the unsteadiness.

The standard deviation (SD) in the four-hole pyramid probe pressure measurements $\left(P_{a}, P_{b}, P_{c}\right.$, and $\left.P_{d}\right)$ was evaluated from the 156 measurements in a constant flow condition $(11.57 \mathrm{~m} / \mathrm{s})$ and was $1.88,5.90,5.68$ and $5.69 \mathrm{~Pa}$, respectively. As the processing uses differential voltages through a common A/D converter, it is not necessary to consider bias errors. The fluctuation in pressure measurements resulted in a $95 \%$ confidence interval for velocity magnitude measurement of range $0.40 \mathrm{~m} / \mathrm{s}(\mathrm{SD}=0.20 \mathrm{~m} / \mathrm{s})$. This relates to an uncertainty of $\approx 0.25^{\circ}$ in both pitch and yaw and a maximum uncertainty of $\approx 0.5^{\circ}$ at the most extreme angles of attack (after Kline and McClintock 1953).

Aerodynamic tests were undertaken with 3, 6, 9 and 12 pairs of ribs. For each the measurement was perpendicular to the mainstream flow and situated directly furthest downstream rib. The traverse mechanism and probe body are downstream of the main experiment (Fig. 1). The probe was traversed in $3 \mathrm{~mm}$ steps in both directions across the measurement plane. Pressure data were taken to within $10 \mathrm{~mm}$ of the trailing edge sidewall of the passage, as preliminary numerical simulations showed that velocity gradients were low in this area. Reynolds number was calculated using the dynamic head measured in the settling length of the passage which did not contain ribs.

\subsection{Heat transfer tests}

The second test at each position was a transient heat transfer test. Initial conditions were isothermal throughout. Flow was drawn through the intake and heater mesh, which produced 
Table 1 Material properties and uncertainties used in processing and uncertainty analysis of experimental heat transfer coefficient distributions

\begin{tabular}{llll}
\hline Property & Nominal value & Relative uncertainty & Absolute uncertainty \\
\hline Perspex thermal product $\left(\mathrm{W} / \mathrm{m}^{2} / \mathrm{s}^{0.5} / \mathrm{K}\right)$ & 569 & $\pm 5.1 \%$ & \pm 29 \\
Perspex thermal conductivity $(\mathrm{W} / \mathrm{m} / \mathrm{K})$ & 0.19 & $\pm 0.5 \%$ & \pm 0.0095 \\
Brass thermal conductivity $(\mathrm{W} / \mathrm{m} / \mathrm{K})$ & 109 & $\pm 5 \%$ & \pm 5.5 \\
Brass density $\left(\mathrm{kg} / \mathrm{m}^{3}\right)$ & 8490 & $\pm 0.5 \%$ & \pm 420 \\
Uncertainties & & & \pm 0.04 \\
Time $(\mathrm{s})$ & & & \pm 0.3 \\
Temperature $(\mathrm{K})$ & & $1.5-4.0$ \\
Uncertainty due to liquid crystal thickness $(\%)$ & & \\
\hline
\end{tabular}

a rapid change in gas temperature. Consecutive tests added an increasing number ribs upstream of the instrumented ribs.Heat transfer testing was undertaken with 3-12 pairs of ribs. A $30 \mu \mathrm{m}$ coating of a mixture of three narrow-band (full activation over $1^{\circ} \mathrm{C}$ ) crystals, and black ink was applied to the suction surface and leading edge of the instrumented area (most downstream 3 rib pairs). Three crystals (nominally displaying peak intensity at 35,40 and $45^{\circ} \mathrm{C}$ ) provide information across the full range of expected surface temperatures, reducing uncertainty in the overall heat transfer coefficient distribution. The black ink reduces transmitted light, which improves the reflection characteristics. Two LED warm white strip lights were used to illuminate the rig, and a digital camcorder (Panasonic NV-D527, $720 \times 576$ resolution, frame rate $25 \mathrm{~Hz}$ ) used to record the crystal activation, with the leading edge reflected in a mirror. Spatial resolutions of 4.8 and 2.1 pixels $/ \mathrm{mm}^{2}$ were achieved on the top (ribbed) and side (smooth) surfaces, respectively. The difference is due to the sidewall being reflected in a mirror to allow a single camera to capture both surfaces simultaneously. Blackout material was used around the passage in order to remove reflections from external sources of light. Three hollow brass ribs were permanently attached to this area of the surface to allow the hybrid rib technique to be used (Wang 1991).

A single gas K-type thermocouple at $12 \mathrm{~Hz}$ is used to record the driving gas temperature at the passage centreline at the streamwise position shown in Fig. 12. As ten individual tests are performed, this is equivalent to simultaneously measuring the temperature at ten points in the full passage. The gas temperature was increased in three steps during a single test. The response of the liquid crystal was captured by video camera. Processing of raw experimental data was carried out using semi-automated in-house software (McGilvray and Gillespie 2011). Material properties used are detailed in Table 1.

\subsection{Transient liquid crystal technique}

The transient liquid crystal technique makes use of the temperature-dependent ability of liquid crystals to selectively reflect circularly polarised light to accurately determine the spatially resolved heat transfer coefficient distribution on a surface. Here the flow is established under ambient conditions, and the rig allowed to come to isothermal conditions. At the start of the test the incoming gas temperature is increased rapidly. The velocity through the heater is $8.1 \mathrm{~m} / \mathrm{s}$ leading to a temperature time constant of $0.016 \mathrm{~s}$. The temperature rise at the surface over the full field of interest is then captured by recording a video of the test surface. The colour and changing intensity can both be related to the surface temperature. The surface temperature history is uniquely related to a surface heat transfer coefficient. To calculate spatially resolved heat transfer coefficients, the 1-D heat equation is solved for a semi-infinite flat plate initially at isothermal conditions and subject to a convective heat load at its top surface. This analysis is sufficient, provided Eqs. 5 and 6 are satisfied (Schultz and Jones 1973). In the tests conducted the large-scale, low-speed and stepped input gas temperature ensures that the heat flux gradients are low on the floor of the ribbed passages and the smooth sidewalls. It is only on the ribs where there is substantial 3-D conduction that the technique cannot be applied; the hybrid technique described below is employed.

$\frac{\partial^{2} q}{\partial y^{2}} \ll \frac{\rho c_{p}}{k} \frac{q}{2 t}$

$\frac{\partial^{2} q}{\partial z^{2}} \ll \frac{\rho c_{p}}{k} \frac{q}{2 t}$

The input gas temperature, which is captured at $12 \mathrm{~Hz}$ through a 16-bit A/D card, is filtered to remove noise and is then modelled as a time separated series of ramps (still at $12 \mathrm{~Hz}$ ). The analytical solution of surface temperature rise in response to a ramp in gas temperature for an arbitrary heat transfer coefficient is known (Ireland and Jones 2000), and a predicted surface temperature history can then be created by the superposition of the time offset ramp solutions assuming constant HTC at any surface location. As the same matrix of surface temperature $\times$ HTC $\times$ time is used in the processing of all pixel locations in each rib pitch, this 


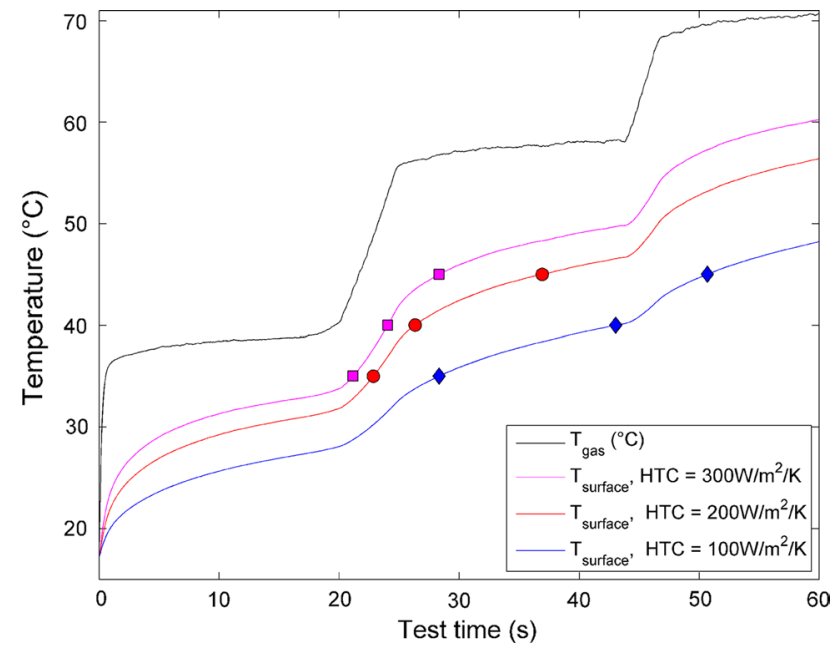

Fig. 8 Experimental gas and projected surface temperature profiles for differing heat transfer coefficients. Symbols indicate time at which $35,40,45^{\circ} \mathrm{C}$ crystals activate for each projected surface temperature history

process is computationally inexpensive and so no downsampling of the initial gas temperature history is required.

The green intensity signal of the liquid crystal coating is monitored during the test, and this can be correlated with the surface temperature using an in situ calibration, performed using non-invasive surface thermocouples. This allowed the calibration to be done with the same configuration of lights and angles as were used during the experimental testing. Uncertainty in the calibration was evaluated due to uncoupled errors in individual measurement quantities, as described below. This was then used in the global error calculation.

During each test, the gas temperature is increased in a number of near step rises (see Fig. 8). This ensures activation of liquid crystals at well-conditioned points in time for all HTC levels. From the analytical solution, surface temperature histories are generated across the full range of expected heat transfer coefficients, converted to green intensity and a global minimisation is used to extract HTC at each pixel location. A spatially resolved map of heat transfer coefficient was then produced and transformed to Nusselt number to eliminate the effect of changes in gas conductivity between tests. A mean value of thermal conductivity of air, $0.0279 \mathrm{~W} / \mathrm{m} / \mathrm{K}$, was applied at all points and all tests to generate the Reynolds number; this introduced insignificant error into the calculation.

The hybrid rib technique (McGilvray and Gillespie 2011), using a lumped heat capacity method, is used to calculate heat transfer through the ribs. Hollow ribs were used to ensure a sufficiently low Biot number $(<0.02)$, which allows a lumped capacitance model to be used.
A perturbation method (Kline and McClintock 1953) was again used to evaluate uncertainty in the heat transfer coefficients reported. Errors were assumed to be independent and of equal probability. Heat transfer experimental uncertainties are shown in Table 1. Total heat transfer coefficient uncertainties were found to be $5.7-7.1 \%$.

\section{Numerical calculation set-up}

The mesh was generated using ANSYS ICEM 14.5 from the experimental geometry, comprising the initial square settling length, 2:1 (horizontal) contraction and 1:2 AR ribbed passage, Fig. 9. The mesh type upon generation was unstructured tetrahedral octree. A grid independence study was carried out to assess the effects of (1) cell size and (2) the tetrahedral to hexahedral conversion. The cross-sectional velocities were assessed at measurement planes behind the first and last (twelfth) ribs. Grid sizes were $3.73 \mathrm{M}$, $5.91 \mathrm{M}$ and $8.81 \mathrm{M}$ cells for the coarse, medium and fine grids, respectively. The medium and fine grids had regions of higher mesh density around the ribs, as shown in Fig. 9. A comparison between three grids is shown in Fig. 10. It can be seen that the contours of velocity for the medium and fine grids are extremely similar; hence, it was chosen to use the medium grid sizing in the heat transfer grid convergence index study below. For the medium and fine grids, a negligibly small difference was also seen in the same velocity contours for the tetrahedral to hexahedral conversion.

A grid convergence index (GCI) study (Roach 1994) was carried out on three meshes, only varying the number of prism layers (the initial volume mesh was kept as generated above). GCI is calculated

$\operatorname{GCI}(\%)=F_{s} \frac{f_{\text {fine }}-f_{\text {medium }}}{f_{\text {fine }}} /\left(r^{p}-1\right) \times 100$

where here $F_{S}$ is a factor of safety $(=1.25), f$ is a solution functional, $r$ is the grid refinement ratio, and $p$ the order of convergence, calculated as

$p=\ln \left(\frac{f_{\text {coarse }}-f_{\text {medium }}}{f_{\text {medium }}-f_{\text {fine }}}\right) / \ln (r)$

where we have used an average value $r=r_{\mathrm{av}}$ between the three grids/two refinements (as the two values are extremely close). The grid convergence index gives an uncertainty due to mesh sizing. An extrapolated value of the functional at zero grid spacing $f_{h=0}$ is calculated from

$f_{h=0} \cong f_{\text {fine }}+\frac{f_{\text {fine }}-f_{\text {medium }}}{r^{p}-1}$

Area-averaged exit temperature from the domain and integral heat flux from the leading edge were assessed, and 


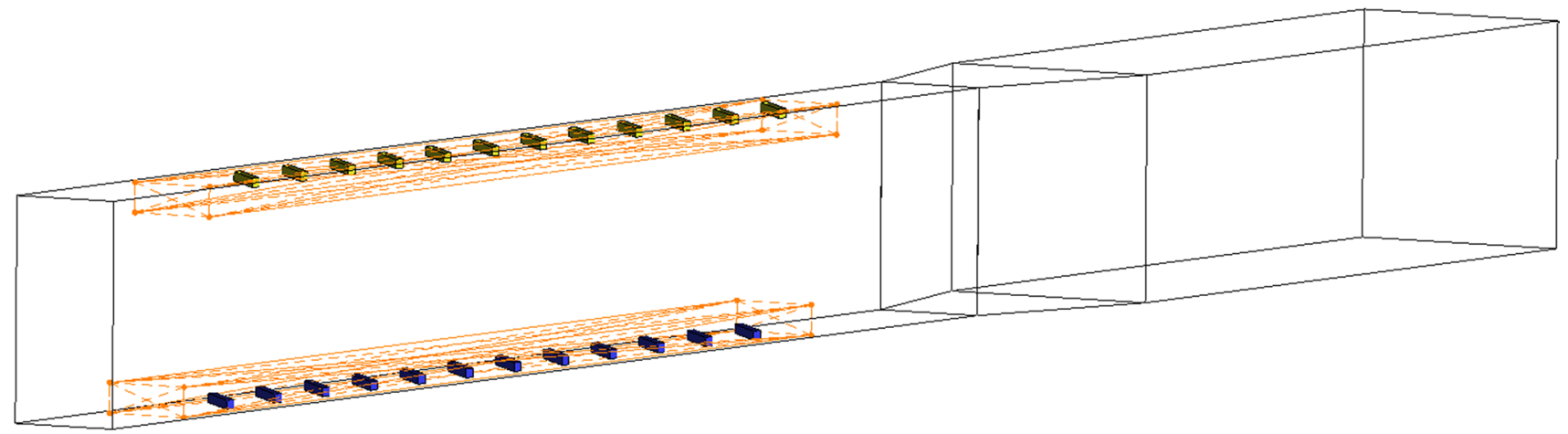

Fig. 9 Computational domain of experimental geometry, showing initial square settling length, 2:1 (horizontal) contraction and ribbed passage. Rib surfaces highlighted, and areas of increased mesh den-
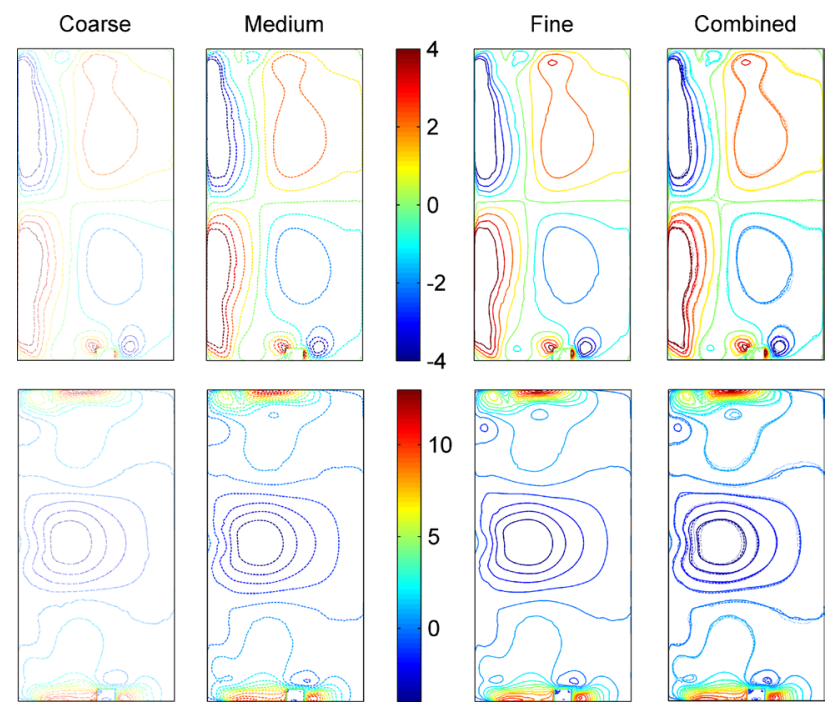

Fig. 10 In-plane/cross-sectional velocities $(\mathrm{m} / \mathrm{s})$ for three grid densities. Top row: vertical velocity (positive up). Bottom row: horizontal velocity (positive left). Same orientation as Fig. 11

the results are summarised in Table 2. Temperature values showed lower uncertainty than the integral heat flux, with both functions showing small values of GCI on the fine grid. This fine grid, used for the simulations below, had 8.08 $\mathrm{M}$ cells $/ 3.53 \mathrm{M}$ nodes, with a highly resolved boundary layer ( 24 prism layers, $\left.r_{e}=1.2, \delta_{1}=5.1 \mu \mathrm{m}\right)$. The 'tet sity (max cell size/ $D_{\mathrm{h}}=0.02$ around ribs indicated. Rest of 1:2 AR passage also at an increased mesh density (max cell size/ $\left.D_{\mathrm{h}}=0.04\right)$. Simulation has 12 pairs of ribs, as below. Flow right to left

to hex' conversion was run following the generation on the boundary layer mesh to reduce the computational expense.

Numerical calculations were performed using ANSYS FLUENT 14.5 for calculations and CFD-Post 14.5 for data extraction. Generation of the Nusselt number distributions was done using MATLAB R2013a. Low Reynolds number, steady calculations were run using the two-equation realisable $k-\epsilon$ model with enhanced wall treatment. The realisable $k-\epsilon$ model was chosen due to its widespread use in industrial practice and its better performance against the $k-\omega$ SST turbulence model for predictions of heat transfer in ribbed cooling passages in previous publications (McGilvray et al. 2013; Pearce 2015). Simulations carried out with $k-\omega$ SST and the Reynolds stress turbulence models showed -5.2 and $+1.1 \%$ difference, respectively, in integral heat flux over the domain respectively when compared to the realisable $k-\epsilon$ simulation. The most significant differences were with the $k-\omega$ SST model, on the ribbed walls $(-6.9 \%)$ and trailing edge $(-12.4 \%)$ in comparison to the realisable $k-\epsilon$ simulation.

Gas density was calculated from the ideal gas equation; specific heat capacity, conductivity and viscosity were calculated from piecewise interpolation of profiles against gas temperature. Spatial discretisation was Green-Gauss node-based for gradient, standard for pressure and secondorder upwind for density, momentum, turbulence kinetic energy, dissipation rate of the turbulence kinetic energy
Table 2 Summary of grid convergence index study

\begin{tabular}{llllllllll}
\hline Mesh & No. of cells $(\mathrm{M})$ & $r\left(r_{\mathrm{av}}\right)$ & $T_{\text {out }}(K)$ & $p$ & $\mathrm{GCI}(\%)$ & $T_{\text {out }, h=0}$ & $Q_{\mathrm{LE}}(W)$ & $\mathrm{GCI}(\%)$ & $Q_{\mathrm{LE}, h=0}$ \\
\hline Coarse & 3.85 & $1.45(1.45)$ & 314.89 & 1.67 & 0.12 & - & 191.88 & 10.01 & - \\
Medium & 5.59 & - & 315.15 & - & - & - & 179.56 & - & - \\
Fine & 8.07 & $1.44(1.45)$ & 315.29 & 1.67 & 0.06 & 315.31 & 177.57 & 1.63 & 175.25 \\
\hline
\end{tabular}

Area-averaged exit temperature and integral heat flux from the leading edge were assessed. $T_{\text {out }, h=0}$ and $Q_{\mathrm{LE}, h=0}$ represent the projected values as the grid spacing tends to zero 
and energy. A coupled pressure-velocity scheme was used. Convergence was obtained for all simulations with residual levels below $10^{-4}$ for continuity, and below $10^{-7}$ momentum, energy and turbulence properties. A constant mass flow rate was set at the inlet plane, with specified turbulence intensity, and length scale from hydraulic diameter. Aerodynamic simulations were carried out at isothermal conditions; the heat transfer simulations with all wall temperatures held at $300 \mathrm{~K}$ and inlet driving gas temperature at $320 \mathrm{~K}$.

\section{Aerodynamic results}

Experimental aerodynamic data are presented as plots of pitch and yaw in Fig. 11a-d where experiments are compared to numerical predictions. Pitch and yaw angles are shown as defined in Fig. 12 The figures show pitch angle (vertical) on the left two maps and yaw angle (horizontal) on the right two maps. The same experimental data are used to form the vector plots seen in the combined aerodynamic and heat transfer images in Fig. 15.

Similar and expected trends in the development of the cross-passage secondary flow are seen in both the experimental and numerical data. Secondary flow vortices are seen in all maps and can be tracked as the development of four main features in pitch and yaw. Flow at large pitch angles was seen along the leading edge (left-hand vertical edge), moving vertically from near the corners towards the centre of this edge. Strong negative yaw is seen in the centre of the passage, becoming less pronounced moving horizontally towards the trailing edge. Along the trailing edge the flow moves from the centre towards the pressure and suction surfaces (ribbed surfaces). Finally the secondary flow close to the pressure and suction surfaces is characterised by positive yaw, returning this flow towards the leading edge of the passage. This main feature is barely apparent downstream of three rib pairs, with the centre of rotation close to the junctions of the leading edge and ribbed surfaces. As the flow develops, the centre of rotation moves onto the centreline of the passage, horizontally, and approximately equidistant from the ribbed end walls. The flow field is clearly close to being fully developed in Fig. 11c, d. Not easily visible in these angle plots but seen in the vector plots in Fig. 15 is a reduction in secondary flow magnitude with the addition of three rib pairs (from 9 to 12 pairs). Here it is speculated that the development of the secondary flow across the full passage leads to enhanced mixing and CFD studies show that a periodic strengthening and decay of the flow secondary flow field then ensues.

It is well established (Han et al. 2000) that ribs arranged in a parallel as opposed to cross-direction configuration set

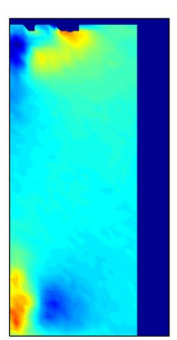

Experimental

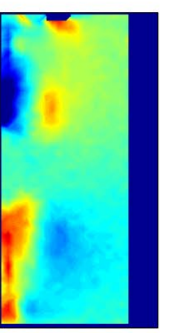

Experimental

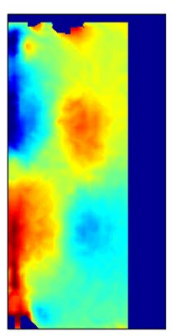

Experimenta

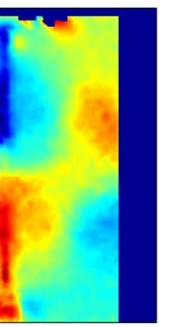

Experimental

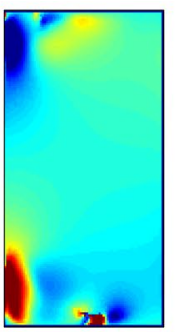

Simulation

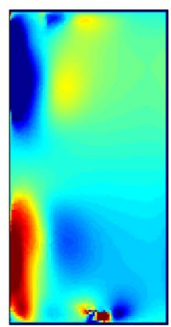

Simulation

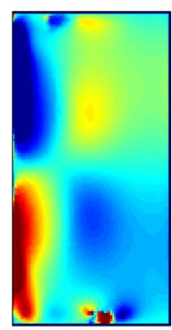

Simulation

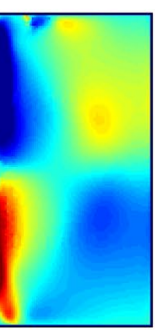

Simulation

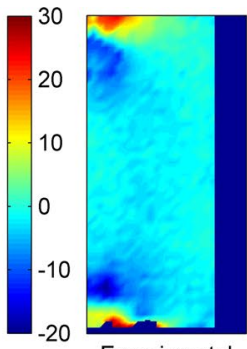

Experimental

(a)

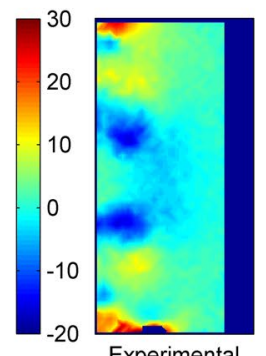

(b)

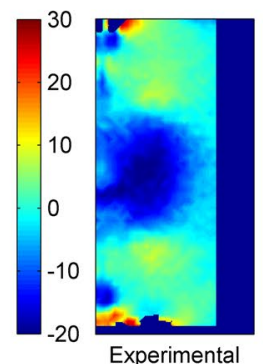

(c)

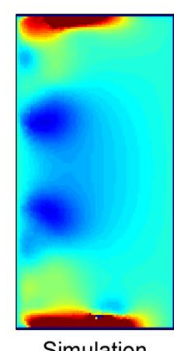

Simulation

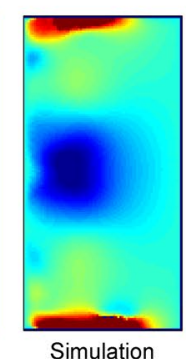

Simulation

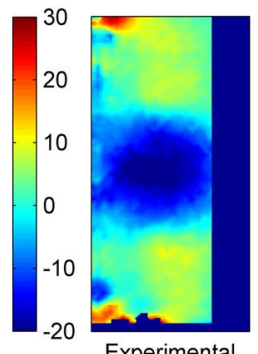

(e)

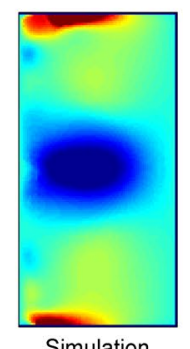

Fig. 11 Experimental and simulation data for increasing numbers of ribs (3, 6, 9, 12 pairs). Left pair of each plot: pitch angle. Right pair of each plot: yaw angle. Edges of each plot (relative position within blade): top: suction surface; right: trailing edge; bottom: pressure surface; left: leading edge. a 3 pairs of ribs. b 6 pairs of ribs. $\mathbf{c} 9$ pairs of ribs. d 12 pairs of ribs

up secondary flows within the flow in the form of counterrotating vortices so it is unsurprising that these are clearly seen in the experimental data; flow driven onto the leading edge by the ribs, then towards the centre of the leading edge, from there it was swept towards the trailing edge as a cross-flow. Other smaller features are also visible. A 


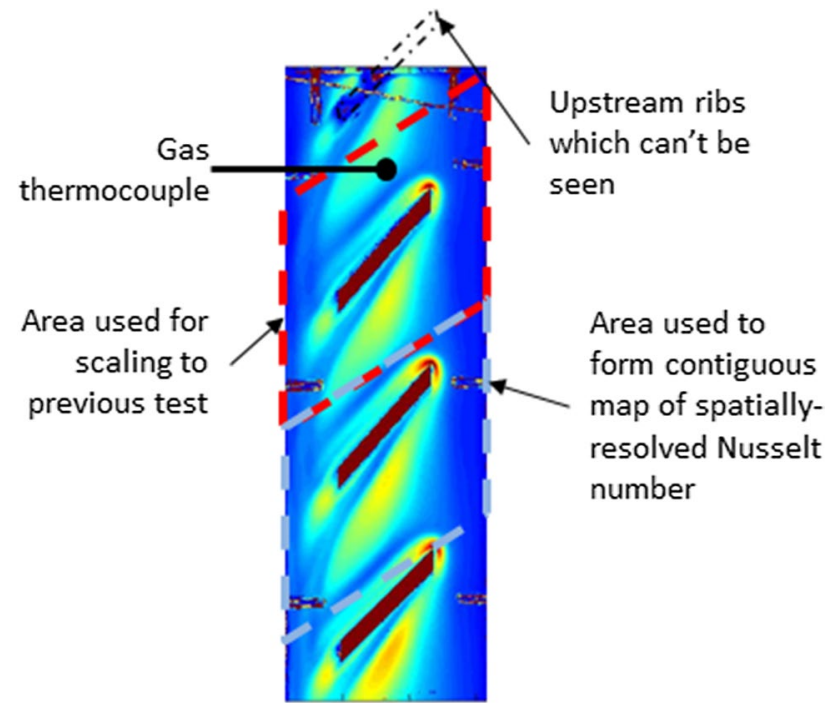

Fig. 12 Areas used in normalisation method of experimental data

pair of vortices develops in the corners of the leading edge, downstream of the end of each rib. These rotate in the same direction as the main secondary vortex in each half of the passage.

\section{Heat transfer results}

Beginning with three fixed pairs of brass ribs, each consecutive test was conducted with an upstream pair of ribs upstream to allow the secondary flow field to develop. Raw experimental data were processed as previously described, and then specific areas from each experiment were normalised and combined in sequence with data from other tests to form a single spatial map of heat transfer coefficient distribution built from small, high-resolution sections.

Normalisation of consecutive experiments was undertaken to allow concatenation of data. A single test gathered data from an area around three ribs (equivalent to ribs 1-2-3 in the first test and 10-11-12 in last) for both the suction surface and leading edge. The data of interest were always taken to surround the middle rib of each test; in particular, the final rib data were excluded to ensure the reattachment of flow to the ribbed surface was accurately captured. An area representing the repeating rib unit was defined for the purposes of normalisation, shown on the experimental data in Fig. 12. A check could be made of the consistency of the data by comparing the middle rib distribution from one spatial position test to the furthest upstream rib in the next test (with an additional rib added). Where there were slight discrepancies seen test to test (area-averaged differences of $8.4 \%$ on the ribbed surface and $5.8 \%$ on the smooth surface), linear scaling was applied to match the average
Nusselt number over equivalent ribs. These differences can be linked to variations in conditions test to test and slight differences in the area of pixels selected for processing from each experimental video. The data of interest surrounding the middle rib were then extracted and concatenated in series to form a spatial Nusselt number map for the full passage. Note that because the ribs are staggered on either side of the passage, a diagonal discrepancy is seen across the leading edge surface. This arises from the use of a single centreline driving gas temperature over the entirety of each rib pitch in the generation of the heat transfer coefficient/ Nusselt number. When averaged, this is typical of the methodology used in analyses by engine designers.

The experimental concatenated map of Nusselt number is presented in Fig. 13. This gives a clear indication of the development of the heat transfer distribution as number of ribs is increased (equivalent to the flow entering and moving along the passage).

Similar heat transfer features to those described in the open literature are seen in the experimental data. On the ribbed wall, high areas of Nusselt number around the upstream corner of the rib due to the generation of a horseshoe vortex as the oncoming turn around the rib. The mainstream flow passing over the ribs reattachs downstream causing triangular regions of high Nusselt number between them. Finally, on the floor of the passage in proximity to the downstream corner of the rib an additional enhancement in Nusselt number is seen. This is associated with a rolled up vortex travelling along the angled rib which then scrubs the passage floor in this location.

The secondary flow induced clearly runs up onto the leading edge of the cooling passage. Here the increased velocity and thinned boundary layer promote high heat transfer. As expected from the aerodynamic measurements, this feature strengthens moving along the passage, plateauing as the flow becomes fully developed and then slightly decreasing in extent.

While the highest Nusselt number would be expected at the entrance of a smooth passage, here precisely the opposite is observed, and the average Nusselt number for the suction surface and leading edge against axial position, shown in Fig. 14, is found to be 1.4-1.5 times higher at the 12th rib pair than for the first, averaging across the ribbed and smooth wall. It is clear that the flow develops substantially over the first 5-6 rib pitches. The difference in level of Nusselt number is stark and emphasises the importance of fully characterising the distribution in the developing flow region.

The results of a CFD simulation of the entire passage are included for comparison. Nusselt number is calculated by Eq. 10, where $x$ is the streamwise position.

$N u_{(x, y, z)}=\frac{\dot{q}_{(x, y, z)} D_{\mathrm{h}}}{k\left(T_{\mathrm{gas}(x)}-T_{\text {wall }}\right)}$ 
Fig. 13 Top experimental Nusselt number distribution (suction surface and leading edge only). Middle simulation Nusselt number distribution (all surfaces). Bottom gas temperature profile from simulation, showed as continuous (blue), and as sampled to match the experimental method (stepped, red)
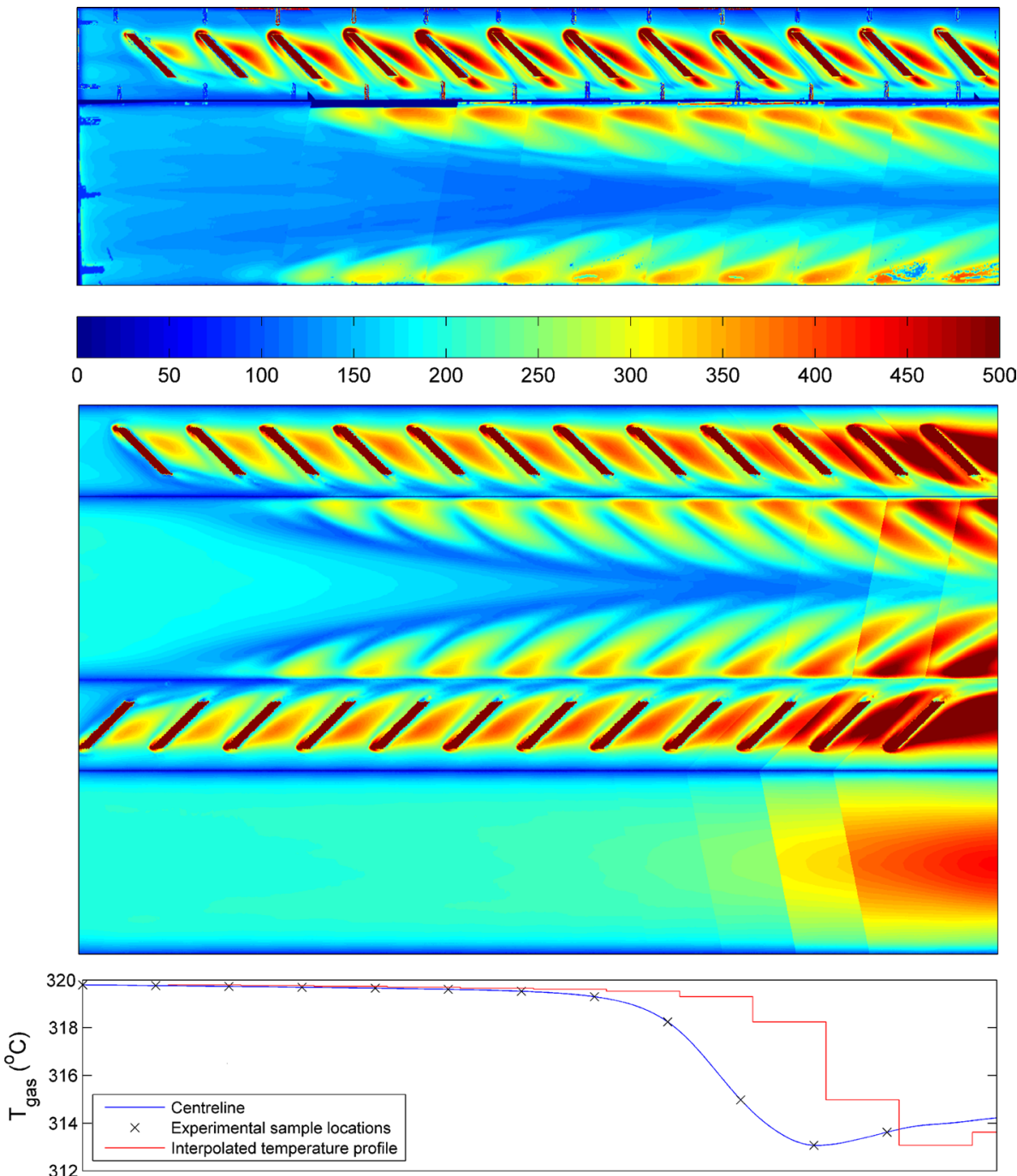

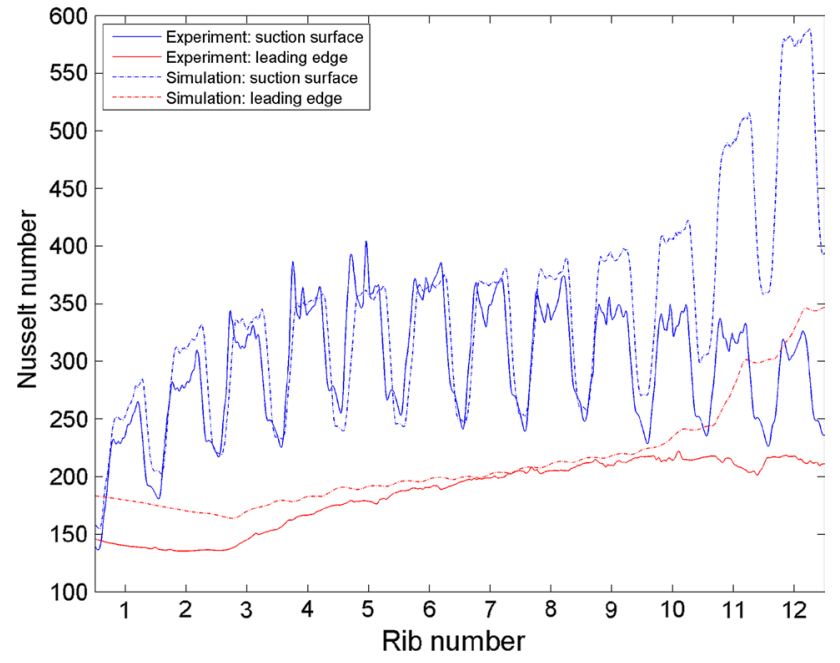

Fig. 14 Circumferentially averaged Nusselt number against axial position for suction surface and leading edge
Again the pronounced discontinuity in $\mathrm{Nu}$ level at the passage exit is caused by discrete steps in driving gas temperature. The CFD illustrates that the level of Nusselt number is similar on the opposing ribbed wall for this stationary passage, while the other smooth wall has substantially lower Nusselt number as it is not subject to the secondary flow wash generated by the parallel rows of ribs. To make a more direct comparison to the experimental data the simulation Nusselt number distribution was calculated for each strip (as in the experiment) based on the centreline gas temperature at the same distance upstream of the rib pitch of interest, exactly as done for the experimental data, Fig. 12. This can be seen in the bottom plot of Fig. 13. This figure indicates that the gas centreline temperature stays fairly constant up until the ninth rib pitch, where it starts to fall significantly, followed by a small recovery at the twelfth rib. This is an accurate prediction of the physical trend seen in experiments instrumented with 
a full distribution of streamwise centreline thermocouples (McGilvray et al. 2013). Whereas the flow has been losing driving potential continuously along the passage, the nature of developing secondary flow-the upper and lower passage vortices-has the effect of rolling the lower cooling potential flow into two regions above and below the centreline, but driving the higher cooling potential flow near the centreline towards the sidewall, thus driving the cooling process. When the vortices have grown such that they occupy the entire cooling passage, lower cooling potential flow must exist at the centreline. This flow is swept across the passage from leading to trailing edge, driving the sudden and progressive drop in centreline gas temperature ( $4 \mathrm{~K}$ from the original driving gas temperature difference of $20 \mathrm{~K}$ ). The mixing of the vortices has the effect of weakening their structure, and rate of temperature drop becomes less pronounced. In the steady numerical calculations two features account for the poorer match to the experimental data in this area. First the CFD mesh is optimised to capture near wall viscous effects; thus, important mixing events near the centre of the passage, which in any case are likely to be highly unsteady, are not well captured by the mid-passage-resolved mesh. Sensible mesh refinement, however, did not improve this substantially. Second, in the current methodology where there has been a one pitch offset in the location of the gas thermocouple relative to most of the solution, the solution is very sensitive to the position at which the centreline temperature first begins to drop, and this leads to large, discrete pitch to pitch variation in this region, over-predicting the HTC in areas of increasing temperature and under-predicting where the temperature is dropping. It is the segregation of colder flow into the secondary flow structures upstream of the 9th rib that contributes to a remarkably constant centreline temperature in that region, and this in turn leads to excellent agreement between the experimental HTCs and CFD predictions. While the HTC could be formed based on the local adiabatic wall temperature to great effect, this would be of little value to an engine designer whose predictive capabilities generally depend on conducting an energy balance along a cooling passage in the absence of known profiles in the cooling flow.

\subsection{Experimental to numerical simulation comparisons}

A qualitative comparison between the experimental and numerical simulation data shows that the main heat transfer features are picked up and the level of Nusselt number is of the same order. The circumferentially averaged values are presented in Fig. 14. The simulated results are particularly good for the first 9-10 rib pitches, where the simulated $\mathrm{Nu}$ vales are on average only $7.6 \%$ above the experimental. Rib pitches 11 and 12 show a significant deviation between experimental and simulation data, the latter predicting an average $\mathrm{Nu} 50.9 \%$ higher than seen in the experiments. The simulation over-predicts Nusselt numbers by $17 \%$ on average.

If the continuous centreline gas temperature profile is used, rather than the interpolated profile method, a significant discrepancy between experimental and CFD Nusselt number maps still exists, though the discontinuities disappear. This indicates that use of the centreline gas temperature for the calculation of HTC may not be the most appropriate value, though this is the standard practice within the field. Gillespie et al. (1994) discuss using mixed bulk temperatures for the calculation of HTC as a more useful, accurate and comparable way of defining HTC.

The experimental difficulties with taking spatially quantified measurements of mixed bulk temperature (mass flux averaged temperature, Eq. 11) could be solved by using the passage build up technique described in this paper. To gather spatially resolved temperature measurements, a coarse grid of fine wire, cross sectional across the passage just downstream of the aerodynamic measurement plane, would be used to hold an array of thermocouples. Streamwise velocity and static pressure are calculated from the aerodynamic measurements. Density can be calculated from the combination of the data sets. Specific heat capacity is either correlated against temperature or assumed constant for an individual cross section.

$T_{m, b}=\frac{\int \rho u c_{p} T d A_{x}}{\int \rho U c_{p} d A_{x}}$

\section{3-D combination plots}

In Fig. 15 the experimental aerodynamic and experimental Nusselt number data are shown combined at the four aerodynamic measurement planes (behind the 3rd-6th-9th12th rib pairs). Now the aerodynamic data are presented in the form of vector plots, showing in-plane directions and magnitude of these velocities, allowing their magnitude to be better understood. The vector plots are scaled to the same base value for both experimental and numerical. The Nusselt number scale is shown in Fig. 13. These plots clearly indicate the fluid dynamical secondary flow features within the bulk flow, but importantly also where the numerical simulation is not able to correctly capture the physics. We can show that the simulations as carried out using industry standard practices and models, while able to produce superficially similar heat transfer distributions, are not in fact really capturing the true aerodynamic mechanisms by which the secondary flow drives the heat transfer distribution observed. This helps explain the substantial differences seen in the last two ribs in Fig. 14.

The combination of aerodynamic and heat transfer data clearly shows the dominant effect of the secondary 


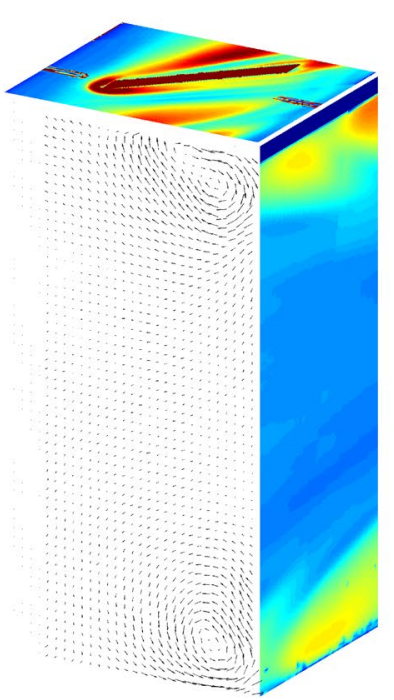

3 ribs

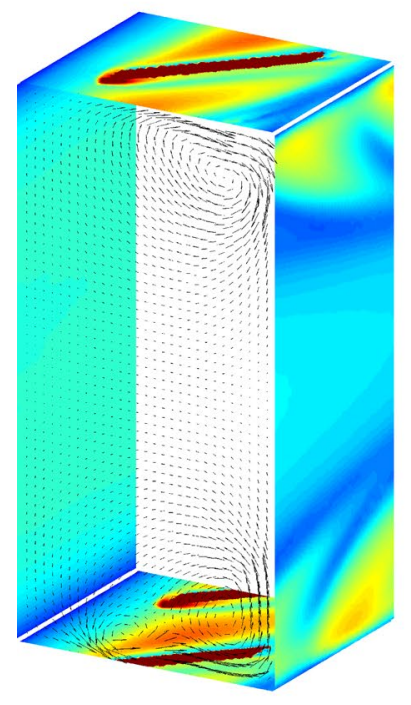

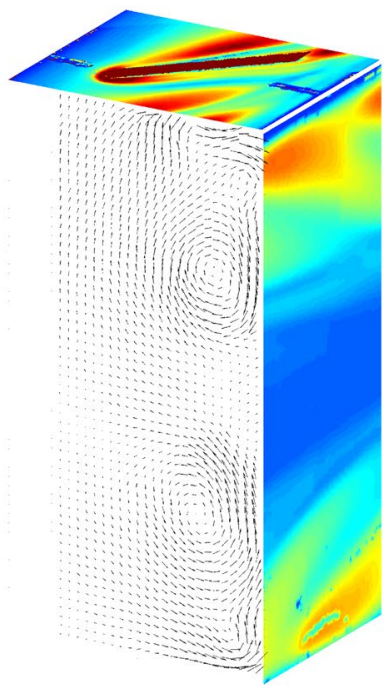

6 ribs

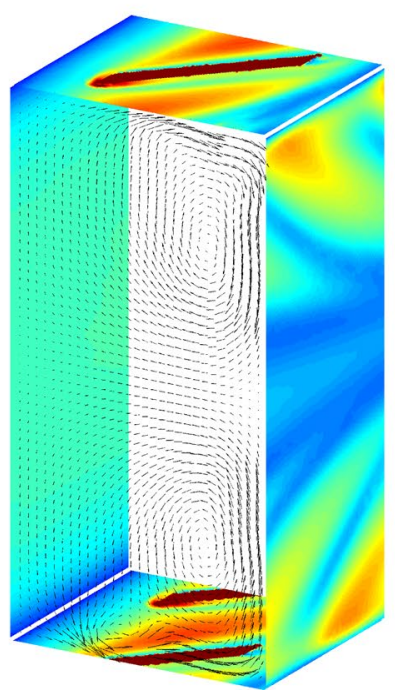

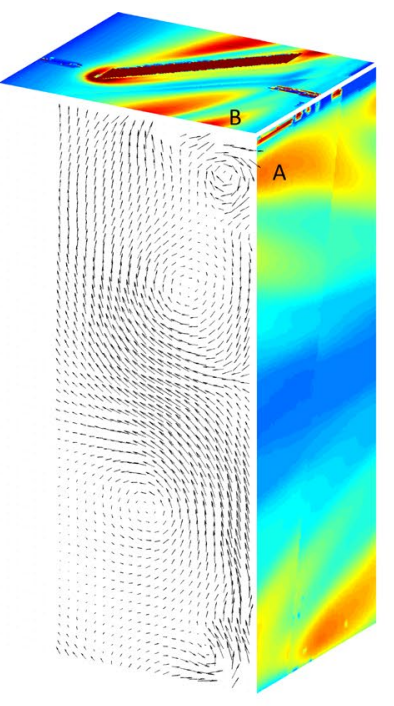

9 ribs

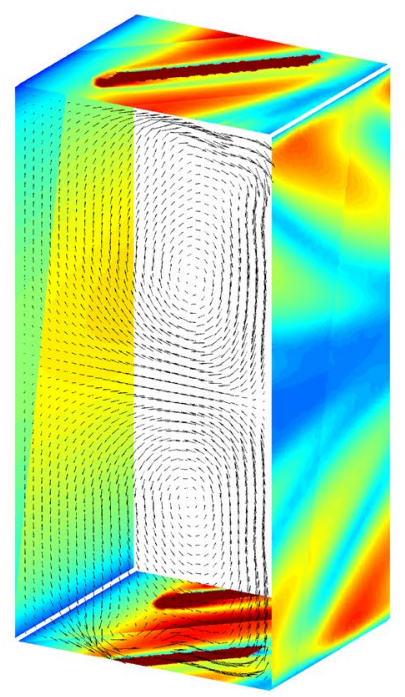

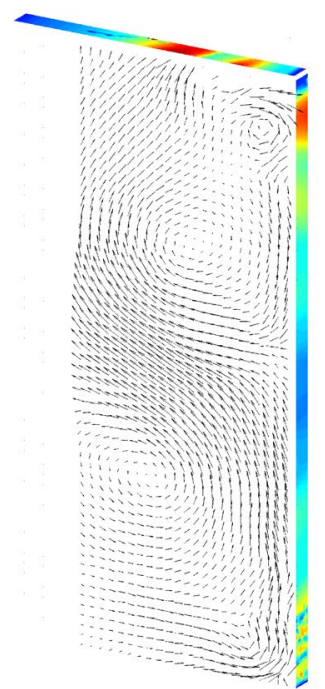

12 ribs

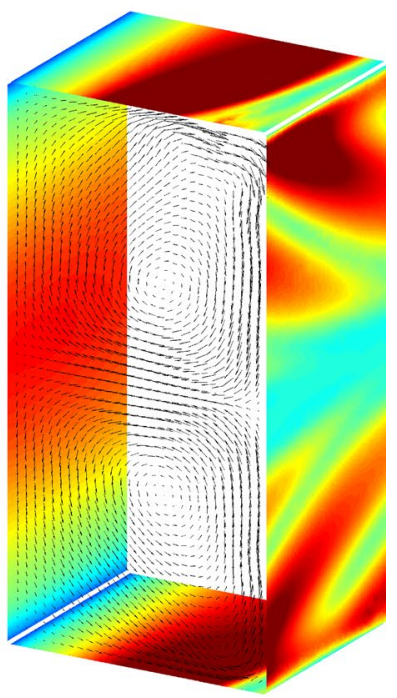

Fig. 15 Three-dimensional combination plots showing aerodynamic cross sections with Nusselt number distribution. The cross section is shown at the most upstream point of each image. Top row experimental. Bottom row numerical simulations. Scale as Fig. 13

flow features in promoting regions of high heat transfer. High heat transfer is seen where the flow impinges onto passage walls, causing a thin thermal boundary layer. Significantly lower heat transfer is observed in regions where flow moves parallel to, or away from the walls (in the cross-sectional plane). The salient aerodynamic and heat transfer features are well matched spatially. In particular in the experimental data (top row of plots) on the suction surface (top surface of each plot) the two regions of high heat transfer in the middle where impingement is seen, and on the leading edge (right side) the small regions of high heat transfer at the extremities where impingement is again seen. Also the region of low heat transfer in the middle of the leading edge, where the flow is seen to move parallel to or away from the surface.
The significance of small secondary flow features can be seen most easily in the case of nine pairs of ribs. Here the small additional corner vortex causes impingement of flow onto the leading edge sidewall and onto a region immediately downstream of the back corner of the rib. These generate high heat transfer. The flow separation from the centre of the leading edge is associated with lower heat transfer. The secondary flow field seen for 9 and 12 pairs of ribs is very similar in structure. Note that only a small area of HTC data was available for the experimental 12 ribs case, hence the short axial distance in the plot.

These combination plots demonstrate that even for the first nine ribs, where matching between the experimental and simulated Nusselt number distributions is very good, the turbulence modelling is not fully capturing the physics 
of the secondary flows. This is primarily as the simulation shows a single pair of counter-rotating vortices in the passage, whereas the experimental data show the development of a second pair of vortices in the passage from six ribs pairs onwards. It appears to be these features that drive the flare of high heat transfer seen on the leading edge sidewall (marked 'A' on experimental nine-rib case, Fig. 15). Experimental data show the flare being comprised of two parts a small, high Nusselt number core, surrounded by a larger, lower Nusselt number outer region. The 3-D combination plots show a strong correlation between the location of the smaller corner vortices and the spots of higher heat transfer in the leading edge flares. This differs from the simulation data, where the majority of the sidewall flare is at a high level, and notably the angle of the flare is more aligned with the mainstream flow in the experiment. The simulation aerodynamic data show no evidence of the smaller vortices, but rather a large sweeping flow from the rib onto the leading edge. This suggests that the level of mixing of this flow with the mainstream is lower and it penetrates further across the passage.

The feature marked as ' $\mathrm{B}$ ' on the same plot is driven by a complex set of interaction of vortices in the passage near to the rib and is simply absent from the simulated data. While numerically the Nusselt number values are superficially similar, the mechanisms by which these features are formed are not correctly modelled in parts the steady RANS CFD, and there must therefore be some risk in extending the modelling to conditions which cannot be validated. This underlines the importance of combining the aerodynamic and heat transfer data when validating numerical modelling.

\section{Conclusions}

An experimental technique has been developed and applied to allow rapid, detailed aerodynamic and heat transfer measurements to be taken in a stationary internal cooling passage with an arbitrary distribution of ribbed turbulators. The modified approach, where the geometry is progressively built, has been shown to generate full surface Nusselt number and allow non-invasive secondary flow field distributions to be measured with high resolution. The inherent error introduced by this strategy was explored numerically and data excluded from analysis appropriately where there are not sufficient geometric features to ensure representative flow.

These data show clear links between small secondary flow features and local Nusselt number enhancement on the passage surface. It has been demonstrated that a full passage Nusselt number map at high resolution can be formed using a piecewise approach, and that heat transfer from developing flow at the passage entrance on a smooth wall is 1.4-1.5 times higher at the last (12th) rib than at entry. Enhancement on the ribbed wall is similar; however, a peak is found after five rib pitches (again approximately 1.5 times the level at the passage entrance); however, this drops to 1.4 towards the passage exit. The implications of this distribution are not clear as the driving gas temperature also drops form the entrance of the passage.

The data have been used for the validation of CFD employing industry best practice meshing and solution strategies. While generally the CFD models the system well, some key flow features responsible for local heat transfer enhancement in real engine cooling geometries, where ribs may not span the full passage, are missed. The 3-D plots combining aerodynamic cross-sectional vector plots with spatially resolved surface Nusselt number maps are then used to investigate the differences between the Nusselt number distributions in these regions. The whole passage Nusselt number was over-predicted by $17 \%$ on the experimentally measured surfaces.

Acknowledgements Thanks to Josh Ryley for his general assistance; also to Duncan Blake and Gerald Walker for both the manufacture and instrumentation of the model. Funding was provided by Seventh Framework Programme (Grant No. FP7/2007-2013).

Open Access This article is distributed under the terms of the Creative Commons Attribution 4.0 International License (http://creativecommons.org/licenses/by/4.0/), which permits unrestricted use, distribution, and reproduction in any medium, provided you give appropriate credit to the original author(s) and the source, provide a link to the Creative Commons license, and indicate if changes were made.

\section{References}

Arts T (2006) Aero-thermal characteristics of the flow in the internal cooling channel of a gas turbine blade. In: Associazione Termotecnica Italiana

Casarsa L, Arts T (2005) Investigation of the aerothermal performance of a high blockage rib-roughened cooling channel. J Turbomach 127:580-588

Gillespie DRH, Byerley AR, Ireland PT, Wang Z, Jones TV (1994) Detailed measurements of local heat transfer coefficient in the entrance to normal and inclined film cooling holes. In: Proceedings of ASME Turbo Expo: Turbine Technical Conference and Exposition

Han JC, Ou S, Park JS, Lei CK (1989) Augmented heat transfer in rectangular channels of narrow aspect ratios with rib turbulators. Int J Heat Mass Transf 32(9): 1619-1630

Han JC, Dutta S, Ekkad V (2000) Gas turbine heat transfer and cooling technology. Taylor and Francis, Oxford

Horlock JH, Watson DT, Jones TV (2001) Limitations on gas turbine performance imposed by large turbine cooling flows. J Eng Gas Turb Power 123:478-494

Iacovides H, Launder BE (2007) Internal blade cooling: the Cinderella of computational and experimental fluid dynamics research in gas turbines. J Power Energy 221(3):265-290

Ireland PT, Jones TV (2000) Liquid crystal measurements of heat transfer and surface shear stress. Meas Sci Technol 11(7):969-986 
Kline S, McClintock FA (1953) Describing uncertainties in singlesample experiments. Mech Eng 75(1):3-8

Kreith F, Boehm RF (2000) Heat and mass transfer: the CRC handbook of thermal engineering. CRC Press LLC, Boca Raton

Kwan PW (2011) Flow management in heat exchanger installations for intercooled turbofan engines. Ph.D. thesis. University of Oxford

Lau SC, Kukreja RT, McMillin RD (1991) Effects of V-shaped rib arrays on turbulent heat transfer and friction of fully developed ow in a square channel. Int J Heat Mass Transf 34(7):1605-1616

Liou TM, Wu YY, Chang Y (1993) LDV measurements of periodic fully developed main and secondary flows in a channel with ribdisturbed walls. J Fluid Eng T ASME 115(1):109-114

Main AJ, Day CRB, Lock GD, Oldfield MLG (1996) Calibration of a four-hole pyramid probe and area traverse measurements in a short-duration transonic turbine cascade tunnel. Exp Fluids 21(4):311

McGilvray M, Gillespie DRH (2011) Transient heat transfer analysis code for liquid crystal experiments at the University of Oxford: updated GUI Driven Software

McGilvray M, Orozco Pineiro C, Axe T, Ryley J, Gillespie DRH (2013) Comparison of stationary internal cooling passage numerical simulations to experimental data (B170). In: 10th European conference on turbomachinery, fluid dynamics and thermodynamics

Pearce R, Ireland PT, He L, McGilvray M, Romero E (2015) Computational and experimental study of heat transfer in rotating ribbed radial turbine cooling passages. In: Proceedings of ASME Turbo Expo: Turbine Technical Conference and Exposition

Rau G, Cakan M, Moeller D, Arts T (1998) The effect of periodic ribs on the local aerodynamic and heat transfer performance of a straight cooling channel. J Turbomach 120:368-375

Roach PJ (1994) Perspective: a method for uniform reporting of grid refinement studies. J Fluids Eng 116(3):405-413

Ryley JC, McGilvray M, Gillespie DRH (2013) Stationary internal cooling passage experiments for an engine realistic configuration (B161). In: Proceedings of the 10th European conference on turbomachinery

Saha K, Acharaya S (2011) Effect of entrance geometry on heat transfer in a narrow $(\mathrm{AR}=1: 4)$ rectangular two pass channel with smooth and ribbed walls (GT2011-46076). In: Proceedings of ASME Turbo Expo 2011, Vancouver

Schultz DL, Jones TV (1973) Heat transfer measurements in short duration hypersonic facilities. AGARDograph No. 165

Tanda G (2010) Effect of rib spacing on heat transfer and friction in a rectangular channel with $45^{\circ}$ angled rib turbulators on one/two walls. Int J Heat Mass Transf 54(6):1081-1090

Tsang CLP, Oldfield MLG (1996) Oxford 4-hole pressure probe

Wang Z (1991) The application of thermochromic liquid crystals to detailed turbine blade cooling measurements. In: D.Phil. thesis, University of Oxford 